\title{
On Solving of Constrained Convex Minimize Problem Using Gradient Projection Method
}

\author{
Taksaporn Sirirut and Pattanapong Tianchai $\mathbb{D}$ \\ Faculty of Science, Maejo University, Chiangmai 50290, Thailand \\ Correspondence should be addressed to Pattanapong Tianchai; pattana@mju.ac.th
}

Received 16 May 2018; Accepted 13 August 2018; Published 1 October 2018

Academic Editor: Jean Michel Rakotoson

Copyright (C) 2018 Taksaporn Sirirut and Pattanapong Tianchai. This is an open access article distributed under the Creative Commons Attribution License, which permits unrestricted use, distribution, and reproduction in any medium, provided the original work is properly cited.

\begin{abstract}
Let $C$ and $Q$ be closed convex subsets of real Hilbert spaces $H_{1}$ and $H_{2}$, respectively, and let $g: C \longrightarrow \mathbb{R}$ be a strictly real-valued convex function such that the gradient $\nabla g$ is an $1 / L$-ism with a constant $L>0$. In this paper, we introduce an iterative scheme using the gradient projection method, based on Mann's type approximation scheme for solving the constrained convex minimization problem (CCMP), that is, to find a minimizer $q \in C$ of the function $g$ over set $C$. As an application, it has been shown that the problem (CCMP) reduces to the split feasibility problem (SFP) which is to find $q \in C$ such that $A q \in Q$ where $A: H_{1} \longrightarrow H_{2}$ is a linear bounded operator. We suggest and analyze this iterative scheme under some appropriate conditions imposed on the parameters such that another strong convergence theorems for the CCMP and the SFP are obtained. The results presented in this paper improve and extend the main results of Tian and Zhang (2017) and many others. The data availability for the proposed SFP is shown and the example of this problem is also shown through numerical results.
\end{abstract}

\section{Introduction}

Throughout this paper, we always assume that $C$ be a closed convex subset of a real Hilbert space $H$ with inner product and norm are denoted by $\langle\cdot, \cdot\rangle$ and $\|\cdot\|$, respectively. Let $g$ : $C \longrightarrow \mathbb{R}$ be a strictly real-valued convex function.

Consider the following constrained convex minimization problem (CCMP):

$$
\min _{x \in C} g(x) .
$$

Assume that (1) is consistent (that is, the CCMP has a solution) and we use $\mathcal{U}$ to denote its solution set. If $g$ is Fréchet differentiable, then the gradient projection algorithm (GPA) is usually applied to solving the CCMP (1), which generates a sequence $\left\{x_{n}\right\}$ through the recursion:

$$
x_{n+1}=P_{C}(I-\lambda \nabla g) x_{n}, \quad \forall n=0,1,2, \ldots,
$$

or more generally,

$$
x_{n+1}=P_{C}\left(I-\lambda_{n} \nabla g\right) x_{n}, \quad \forall n=0,1,2, \ldots,
$$

where the initial guess $x_{0} \in C$ is chosen arbitrarily, the parameters $\lambda$ or $\lambda_{n}$ are positive real number, and $P_{C}$ is the metric projection from $H$ onto $C$. It is well known that the convergence of algorithms (2) and (3) depends on the behavior of the gradient $\nabla g$. It is known from Levitin and Polyak [1] that if $\nabla g$ is $\alpha$-strongly monotone and $L$ Lipschitzian, that is, there exists the constants $\alpha>0$ and $L>0$ such that

$$
\langle\nabla g(x)-\nabla g(y), x-y\rangle \geq \alpha\|x-y\|^{2}, \quad \forall x, y \in C,
$$
and

$$
\|\nabla g(x)-\nabla g(y)\| \leq L\|x-y\|, \quad \forall x, y \in C,
$$

respectively, then, for $0<\lambda<2 \alpha / L^{2}$, the operator

$$
T=P_{C}(I-\lambda \nabla g)
$$

is a contraction; hence, the sequence $\left\{x_{n}\right\}$ defined by the GPA (2) converges in norm to the unique minimizer of the CCMP (1). More generally, for $0<\lambda_{n}<2 \alpha / L^{2}$ for all $n=0,1,2, \ldots$, the operator

$$
G_{n}=P_{C}\left(I-\lambda_{n} \nabla g\right)
$$


is a contraction; if the sequence $\left\{\lambda_{n}\right\}$ is chosen satisfying the property

$$
0<\liminf _{n \longrightarrow \infty} \lambda_{n} \leq \limsup _{n \rightarrow \infty} \lambda_{n}<\frac{2 \alpha}{L^{2}},
$$

then the sequence $\left\{x_{n}\right\}$ defined by the GPA (3) converges in norm to the unique minimizer of the CCMP (1). However, if the gradient $\nabla g$ fails to be $\alpha$-strongly monotone (it means that the gradient $\nabla g$ only satisfies the $L$-Lipschitzian condition), then the operators $T$ and $G_{n}$ defined by (6) and (7), respectively, may fail to be contraction; consequently, the sequence $\left\{x_{n}\right\}$ generated by algorithms (2) and (3) may fail to converge strongly (see also Xu [2]) in the setting of infinitedimensional real Hilbert space, but still converge weakly as the following statement.

Theorem 1 (see $[1,2]$ ). Assume that the CCMP (1) is the unique consistent. Let the gradient $\nabla g$ satisfy the LLipschitzian condition and the sequence of the parameter $\left\{\lambda_{n}\right\}$ satisfies the following condition:

$$
0<a \leq \lambda_{n} \leq b<\frac{2}{L},
$$

for all $n=0,1,2, \ldots$, where $a$ and $b$ are the constants. Then the sequence generated by the GPA (3) converges weakly to the minimizer of the CCMP (1). Indeed, the results of this theorem still hold on the gradient $\nabla g$ which satisfies an $1 / L$-inverse strongly monotone with $L>0$ (in brief, we denote $1 / L$-ism), that is, $\langle\nabla g(x)-\nabla g(y), x-y\rangle \geq(1 / L)\|\nabla g(x)-\nabla g(y)\|^{2}$ for all $x, y \in C$, because the class of L-Lipschitzian mapping contains the class of $1 / L$-ism mapping.

We observe from Theorem 1 that if the parameter $\left\{\lambda_{n}\right\}$ converges to $\lambda \in(0,2 / L)$ such that $\left\{\lambda_{n}\right\}$ satisfies the condition (9) then $q \in C$ solves the CCMP (1) which is the unique consistent if and only if $q$ solves the fixed-point equation

$$
q=P_{C}(I-\lambda \nabla g) q .
$$

It is well known that the gradient-projection algorithm is very useful in dealing with the CCMP (1) and has extensively been studied (see [1-9] and the references therein). It has recently been applied to solve the split feasibility problems (SFP) (see [10-15]) which find applications in image reconstructions and the intensity modulated radiation therapy (see [13-18]). We now consider the following regularized minimization (that is, the CCMP (1) has the unique minimizer solution) problem:

$$
\min _{x \in C} g_{\beta_{n}}(x):=g(x)+\frac{\beta_{n}}{2}\|x\|^{2},
$$

where $\beta_{n}>0$ for all $n=0,1,2, \ldots$ and $g: C \longrightarrow \mathbb{R}$ is a continuous differentiable function, and we also consider the regularized gradient-projection algorithm which generates a sequence $\left\{x_{n}\right\}$ by the following recursive formula:

$$
\begin{aligned}
x_{n+1} & =P_{C}\left(I-\lambda \nabla g_{\beta_{n}}\right) x_{n} \\
& =P_{C}\left(I-\lambda\left(\nabla g+\beta_{n} I\right)\right) x_{n}, \quad \forall n=0,1,2, \ldots
\end{aligned}
$$

Many researchers studied the strong convergency theorems for solving the CCMP (1) using the sequence $\left\{x_{n}\right\}$ which is generated by algorithm (12) for their proposal on the gradient $\nabla g$ which is the class of nonexpansive mapping and the class of $L$-Lipschitzian mapping (see [19-25]) and in case the gradient $\nabla g$ is the class of $1 / L$-ism mapping such that $L>0, \mathrm{Xu}(2010)$ introduced the sequence $\left\{x_{n}\right\}$ which is generated by algorithm (12), and he proved that this sequence $\left\{x_{n}\right\}$ converges weakly to the minimizer of the CCMP (1) in the setting of infinite-dimensional real Hilbert space (see [15]) under some appropriate condition.

Recently, Tian and Zhang (2017) introduced the sequence $\left\{x_{n}\right\}$ generated by algorithm (12), and they proved that this sequence $\left\{x_{n}\right\}$ converges strongly to the minimizer of the CCMP (1) in the same setting of infinite-dimensional real Hilbert space (see [26]) under the control conditions:

(i) $0<\lambda<2 /(L+2)$.

(ii) $\left\{\beta_{n}\right\} \subset(0,1), \lim _{n \rightarrow \infty} \beta_{n}=0$ and $\sum_{n=0}^{\infty} \beta_{n}=\infty$.

(iii) $\sum_{n=0}^{\infty}\left|\beta_{n+1}-\beta_{n}\right|<\infty$.

In this paper, under the motivated and the inspired by above results, we introduce new iterative scheme, based on Mann's type approximation scheme for solving the CCMP (1) in the case of the gradient $\nabla g$ being the class of $1 / L$-ism mapping such that $L>0$ as follows:

$$
\begin{aligned}
& x_{0} \in C, \\
& x_{n+1}=\alpha_{n} x_{n}+\left(1-\alpha_{n}\right) P_{C}\left(I-\lambda\left(\nabla g+\beta_{n} I\right)\right) x_{n}, \\
& \forall n=0,1,2, \ldots,
\end{aligned}
$$

under the mild some appropriate conditions of the parameters $\left\{\alpha_{n}\right\},\left\{\beta_{n}\right\}$, and $\lambda$, we obtain a strong convergency theorem to solve the CCMP (1), in which condition (iii) $\sum_{n=0}^{\infty} \mid \beta_{n+1}-$ $\beta_{n} \mid<\infty$ of Tian and Zhang to be removed. In Section 4 of the applications, it has been shown that the CCMP (1) reduces to the split feasibility problem (SFP) and the data availability for the proposed SFP is shown in Section 5, and the example of this problem is also shown in Section 6 through numerical results.

\section{Preliminaries}

Let $C$ be a nonempty closed convex subset of a real Hilbert space $H$. If $g: C \longrightarrow \mathbb{R}$ is a differentiable function, then we denote $\nabla g$ the gradient of the function $g$. We will also use the notation: $\longrightarrow$ to denote the strong convergency, $\rightarrow$ to denote the weak convergency, and $\operatorname{Fix}(T)=\{x: x=T x\}$ to denote the fixed point set of the mapping $T$.

Recall that the metric projection $P_{C}: H \longrightarrow C$ is defined as follows: for each $x \in H, P_{C} x$ is the unique point in $C$ satisfying

$$
\left\|x-P_{C} x\right\|=\inf \{\|x-y\|: y \in C\} .
$$

Let $g: C \longrightarrow \mathbb{R}$ be a function. Recall that the function $g$ is a strictly real-valued convex function if

$$
\begin{aligned}
g(\lambda x+(1-\lambda) y)<\lambda g(x)+ & (1-\lambda) g(y), \\
& \forall \lambda \in[0,1], \forall x, y \in C,
\end{aligned}
$$


such that $x \neq y$. We collect together some known lemmas and definitions which are our main tool in proving our results.

Lemma 2. Let $H$ be a real Hilbert space. Then, for all $x, y \in H$,

$$
\|x+y\|^{2}=\|x\|^{2}+2\langle x, y\rangle+\|y\|^{2} .
$$

Lemma 3 (see [27]). Let $C$ be a nonempty closed convex subset of a real Hilbert space $H$. Then,

$$
z=P_{C} x \Longleftrightarrow\langle x-z, z-y\rangle \geq 0, \quad \forall x \in H, y \in C .
$$

Definition 4. Let $H$ be a real Hilbert space. The operator $T$ : $H \longrightarrow H$ is called

(i) $L$-Lipschitzian with $L>0$ if

$$
\|T x-T y\| \leq L\|x-y\|, \quad \forall x, y \in H,
$$

(ii) $k$-contraction with a positive real number $k$ such that $k \in(0,1)$ if

$$
\|T x-T y\| \leq k\|x-y\|, \quad \forall x, y \in H,
$$

(iii) nonexpansive if

$$
\|T x-T y\| \leq\|x-y\|, \quad \forall x, y \in H,
$$

(iv) monotone if

$$
\langle T x-T y, x-y\rangle \geq 0, \quad \forall x, y \in H,
$$

(v) $\alpha$-strongly monotone if

$$
\langle T x-T y, x-y\rangle \geq \alpha\|x-y\|^{2}, \quad \forall x, y \in H,
$$

(vi) $\alpha$-inverse strongly monotone $(\alpha$-ism) if

$$
\langle T x-T y, x-y\rangle \geq \alpha\|T x-T y\|^{2}, \quad \forall x, y \in H,
$$

(vii) firmly nonexpansive if

$$
\begin{array}{r}
\|T x-T y\|^{2} \leq\|x-y\|^{2}-\|(I-T) x-(I-T) y\|^{2}, \\
\forall x, y \in H .
\end{array}
$$

Lemma 5 (see [27]). Let $C$ be a nonempty closed convex subset of a real Hilbert space $H$. Then, for $x, y \in C$ and $\alpha \in[0,1]$, we have

$$
\begin{aligned}
\|\alpha x+(1-\alpha) y\|^{2}= & \alpha\|x\|^{2}+(1-\alpha)\|y\|^{2} \\
& -\alpha(1-\alpha)\|x-y\|^{2} .
\end{aligned}
$$

Lemma 6 (see [28]). Let $H$ be a real Hilbert space and $T: H \longrightarrow H$ be an operator. The following statements are equivalent:

(i) $T$ is firmly nonexpansive,

(ii) $\|T x-T y\|^{2} \leq\langle x-y, T x-T y\rangle, \forall x, y \in H$,

(iii) $I-T$ is firmly nonexpansive.
Lemma 7 (see [28]). Let $H$ and $K$ be two real Hilbert spaces and let $T: K \longrightarrow K$ be a firmly nonexpansive mapping such that $\|(I-T) x\|$ is a convex function from $K$ to $\overline{\mathbb{R}}=[-\infty,+\infty]$. Let $A: H \longrightarrow K$ be a bounded linear operator and $f(x)=$ $(1 / 2)\|(I-T) A x\|^{2}$ for all $x \in H$. Then,

(i) $\nabla f(x)=A^{*}(I-T) A x$ for all $x \in H$ where $A^{*}$ is adjoint operator of $A$,

(ii) $\nabla f$ is $\|A\|^{2}$-Lipschitzian.

Lemma 8 (see $[29,30]$ ). Let $C$ be a nonempty closed convex subset of a real Hilbert space $H$. Let $\left\{T_{n}\right\}$ and $\varphi$ be two classes of nonexpansive mappings from $C$ into $C$ such that

$$
\emptyset \neq \operatorname{Fix}(\varphi)=\bigcap_{n=0}^{\infty} \operatorname{Fix}\left(T_{n}\right) .
$$

Then, for any bounded sequence $\left\{z_{n}\right\} \subset C$, we have

(i) if $\lim _{n \rightarrow \infty}\left\|z_{n}-T_{n} z_{n}\right\|=0$ then $\lim _{n \rightarrow \infty}\left\|z_{n}-T z_{n}\right\|=0$ for all $T \in \varphi$, which is called that the NST-condition(I),

(ii) if $\lim _{n \rightarrow \infty}\left\|z_{n+1}-T_{n} z_{n}\right\|=0$ then $\lim _{n \rightarrow \infty} \| z_{n}-$ $T_{m} z_{n} \|=0$ for all $m \in \mathbb{N} \cup\{0\}$, which is called that the NST-condition (II).

Lemma 9 (see [31] (demiclosedness principle)). Let $C$ be $a$ nonempty closed convex subset of a real Hilbert space $H$ and let $S: C \longrightarrow C$ be a nonexpansive mapping with $\mathrm{Fix}(S) \neq \emptyset$. If the sequence $\left\{x_{n}\right\} \subset C$ converges weakly to $x$ and the sequence $\left\{(I-S) x_{n}\right\}$ converges strongly to $y$. Then, $(I-S) x=y$, in particular, if $y=0$ then $x \in \operatorname{Fix}(S)$.

Lemma 10 (see [32]). Let $\left\{a_{n}\right\}$ be a sequence of nonnegative real number such that

$$
a_{n+1} \leq\left(1-\gamma_{n}\right) a_{n}+\gamma_{n} \delta_{n}, \quad \forall n=0,1,2, \ldots,
$$

where $\left\{\gamma_{n}\right\}$ is a sequence in $(0,1)$ and $\left\{\delta_{n}\right\}$ is a sequence in $\mathbb{R}$ such that

(i) $\sum_{n=0}^{\infty} \gamma_{n}=\infty$;

(ii) $\limsup _{n \rightarrow \infty} \delta_{n} \leq 0$ or $\sum_{n=0}^{\infty}\left|\gamma_{n} \delta_{n}\right|<\infty$.

Then, $\lim _{n \rightarrow \infty} a_{n}=0$.

\section{Main Result}

Throughout this paper, we let $C$ be a nonempty closed convex subset of a real Hilbert space $H$. First, we will show that $G_{n}$ which is defined by

$$
\begin{aligned}
& G_{n} x=\alpha_{n} x+\left(1-\alpha_{n}\right) P_{C}(I-\lambda\left.\left(\nabla g+\beta_{n} I\right)\right) x, \\
& \forall x \in C, n=0,1,2, \ldots
\end{aligned}
$$

has the unique fixed point under the conditions $0<\lambda<$ $2 /(L+2), 0 \leq \alpha_{n}<1$ and $0<\beta_{n}<1$ where $g: C \longrightarrow \mathbb{R}$ be a strictly real-valued convex function such that $\nabla g$ is $1 / L$-ism 
with $L>0$. Since, $\nabla g$ is $1 / L$-ism and the nonexpansiveness of $P_{C}$. Then, for each $x, y \in C$, we have

$$
\begin{aligned}
\| P_{C} & \left(I-\lambda\left(\nabla g+\beta_{n} I\right)\right) x-P_{C}\left(I-\lambda\left(\nabla g+\beta_{n} I\right)\right) y \|^{2} \\
\leq & \left\|\left(I-\lambda\left(\nabla g+\beta_{n} I\right)\right) x-\left(I-\lambda\left(\nabla g+\beta_{n} I\right)\right) y\right\|^{2} \\
= & \left\|\left(1-\lambda \beta_{n}\right)(x-y)-\lambda(\nabla g(x)-\nabla g(y))\right\|^{2} \\
= & \left(1-\lambda \beta_{n}\right)^{2}\|x-y\|^{2}+\lambda^{2}\|\nabla g(x)-\nabla g(y)\|^{2} \\
& -2 \lambda\left(1-\lambda \beta_{n}\right)\langle x-y, \nabla g(x)-\nabla g(y)\rangle \\
\leq & \left(1-\lambda \beta_{n}\right)^{2}\|x-y\|^{2}+\lambda^{2}\|\nabla g(x)-\nabla g(y)\|^{2} \\
& -\frac{2}{L} \lambda\left(1-\lambda \beta_{n}\right)\|\nabla g(x)-\nabla g(y)\|^{2} \\
= & \left(1-\lambda \beta_{n}\right)^{2}\|x-y\|^{2} \\
& +\left(\lambda^{2}-\frac{2}{L} \lambda\left(1-\lambda \beta_{n}\right)\right)\|\nabla g(x)-\nabla g(y)\|^{2} \\
\leq & \left(1-\lambda \beta_{n}\right)^{2}\|x-y\|^{2} \\
& +\left(\lambda^{2}-\frac{2}{L} \lambda+\frac{2}{L} \lambda^{2}\right)\|\nabla g(x)-\nabla g(y)\|^{2} \\
= & \left(1-\lambda \beta_{n}\right)^{2}\|x-y\|^{2} \\
& -\lambda\left(\frac{2}{L}(1-\lambda)-\lambda\right)\|\nabla g(x)-\nabla g(y)\|^{2} \\
\leq & \left(1-\lambda \beta_{n}\right)^{2}\|x-y\|^{2} .
\end{aligned}
$$

Therefore,

$$
\begin{aligned}
& \left\|P_{C}\left(I-\lambda\left(\nabla g+\beta_{n} I\right)\right) x-P_{C}\left(I-\lambda\left(\nabla g+\beta_{n} I\right)\right) y\right\| \\
& \quad \leq\left(1-\lambda \beta_{n}\right)\|x-y\| .
\end{aligned}
$$

It follows that, for $x \neq y$, by (30) we have

$$
\begin{aligned}
& \left\|G_{n} x-G_{n} y\right\|=\|\left(\alpha_{n} x\right. \\
& \left.\quad+\left(1-\alpha_{n}\right) P_{C}\left(I-\lambda\left(\nabla g+\beta_{n} I\right)\right) x\right)-\left(\alpha_{n} y\right. \\
& \left.\quad+\left(1-\alpha_{n}\right) P_{C}\left(I-\lambda\left(\nabla g+\beta_{n} I\right)\right) y\right)\|=\| \alpha_{n}(x \\
& \quad-y)+\left(1-\alpha_{n}\right)\left(P_{C}\left(I-\lambda\left(\nabla g+\beta_{n} I\right)\right) x\right. \\
& \left.-P_{C}\left(I-\lambda\left(\nabla g+\beta_{n} I\right)\right) y\right)\left\|\leq \alpha_{n}\right\| x-y \|+(1 \\
& \left.-\alpha_{n}\right) \| P_{C}\left(I-\lambda\left(\nabla g+\beta_{n} I\right)\right) x-P_{C}(I \\
& \left.-\lambda\left(\nabla g+\beta_{n} I\right)\right) y\left\|\leq \alpha_{n}\right\| x-y \|+\left(1-\alpha_{n}\right)(1 \\
& \left.-\lambda \beta_{n}\right)\|x-y\|<\alpha_{n}\|x-y\|+\left(1-\alpha_{n}\right)\|x-y\| \\
& =\|x-y\| .
\end{aligned}
$$

That is,

$$
\left\|G_{n} x-G_{n} y\right\|<\|x-y\|, \quad \forall x \neq y .
$$

So, $G_{n}$ is a contraction, therefore, by Banach's contraction principle, $G_{n}$ has the unique fixed point. Therefore, $G_{n}$ is welldefined.

Let $\mathcal{U}$ be the solution set of the CCMP (1). It is clear that $\mathcal{U}$ is a closed and convex sets. We now ready to present my main results as follows.

Theorem 11. Let $C$ be a nonempty closed convex subset of a real Hilbert space $H, g: C \longrightarrow \mathbb{R}$ is a strictly real-valued convex function such that the gradient $\nabla g$ is $1 / L$-ism with $L>$ 0 . Assume that $\mathscr{U} \neq \emptyset$ and let $\left\{x_{n}\right\} \subset C$ be a sequence generated by

$$
\begin{aligned}
& x_{0} \in C, \\
& x_{n+1}=\alpha_{n} x_{n}+\left(1-\alpha_{n}\right) P_{C}\left(I-\lambda\left(\nabla g+\beta_{n} I\right)\right) x_{n}, \\
& \forall n=0,1,2, \ldots,
\end{aligned}
$$

where $\lambda \in(0,2 /(2+L))$ and $\left\{\alpha_{n}\right\} \subset[0,1),\left\{\beta_{n}\right\} \subset(0,1)$ satisfy the following conditions:

(i) $\alpha_{n} \leq \beta_{n}^{m}$ such that $m>1$ for all $n=0,1,2, \ldots$,

(ii) $\lim _{n \rightarrow \infty} \beta_{n}=0$ and $\sum_{n=0}^{\infty} \beta_{n}=\infty$,

then the sequence $\left\{x_{n}\right\}$ converges strongly to $q \in \mathcal{U}$, which is the unique minimizer of the CCMP (1).

Proof. We divide the proof into 4 steps.

Step 1. We will show that $\left\{x_{n}\right\}$ is bounded. Let $p \in \mathcal{U}$. By the strictly convexity of $g$, we have that $\mathscr{U}$ is a singleton set. Noticing from $1 / L$-ism of $\nabla g$ that $\nabla g$ is $L$-Lipschitzian. So, by (10), we have $p=P_{C}(I-\lambda \nabla g) p$. Therefore, by (30), we have

$$
\begin{aligned}
& \left\|x_{n+1}-p\right\|=\|\left(\alpha_{n} x_{n}\right. \\
& \left.\quad+\left(1-\alpha_{n}\right) P_{C}\left(I-\lambda\left(\nabla g+\beta_{n} I\right)\right) x_{n}\right)-p \| \\
& =\|\left(\alpha_{n} x_{n}+\left(1-\alpha_{n}\right) P_{C}\left(I-\lambda\left(\nabla g+\beta_{n} I\right)\right) x_{n}\right) \\
& -\left(\alpha_{n}+\left(1-\alpha_{n}\right)\right) p\|=\| \alpha_{n}\left(x_{n}-p\right)+\left(1-\alpha_{n}\right) \\
& \cdot\left(P_{C}\left(I-\lambda\left(\nabla g+\beta_{n} I\right)\right) x_{n}-p\right)\|=\| \alpha_{n}\left(x_{n}-p\right) \\
& \quad+\left(1-\alpha_{n}\right)\left(P_{C}\left(I-\lambda\left(\nabla g+\beta_{n} I\right)\right) x_{n}\right. \\
& \left.-P_{C}(I-\lambda \nabla g) p\right)\left\|\leq \alpha_{n}\right\| x_{n}-p \|+\left(1-\alpha_{n}\right) \\
& \cdot\left\|P_{C}\left(I-\lambda\left(\nabla g+\beta_{n} I\right)\right) x_{n}-P_{C}(I-\lambda \nabla g) p\right\| \\
& \quad \leq \alpha_{n}\left\|x_{n}-p\right\|+\left(1-\alpha_{n}\right) \\
& \cdot\left(\| P_{C}\left(I-\lambda\left(\nabla g+\beta_{n} I\right)\right) x_{n}\right. \\
& -P_{C}\left(I-\lambda\left(\nabla g+\beta_{n} I\right)\right) p \| \\
& \left.\quad+\left\|P_{C}\left(I-\lambda\left(\nabla g+\beta_{n} I\right)\right) p-P_{C}(I-\lambda \nabla g) p\right\|\right) \\
& \quad \leq \alpha_{n}\left\|x_{n}-p\right\|+\left(1-\alpha_{n}\right)\left(\left(1-\lambda \beta_{n}\right)\left\|x_{n}-p\right\|\right. \\
& \left.\quad+\left\|\left(I-\lambda\left(\nabla g+\beta_{n} I\right)\right) p-(I-\lambda \nabla g) p\right\|\right)=\alpha_{n} \| x_{n} \\
& -P \|+\left(1-\alpha_{n}\right)\left(\left(1-\lambda \beta_{n}\right)\left\|x_{n}-p\right\|+\lambda \beta_{n}\|p\|\right)
\end{aligned}
$$




$$
\begin{gathered}
=\alpha_{n}\left\|x_{n}-p\right\|+\left(1-\alpha_{n}\right)\left(1-\lambda \beta_{n}\right)\left\|x_{n}-p\right\|+(1 \\
\left.-\alpha_{n}\right) \lambda \beta_{n}\|p\|=\left(\alpha_{n}+\left(1-\alpha_{n}\right)\left(1-\lambda \beta_{n}\right)\right) \| x_{n} \\
-p\left\|+\left(1-\alpha_{n}\right) \lambda \beta_{n}\right\| p\left\|=\left(1-\left(1-\alpha_{n}\right) \lambda \beta_{n}\right)\right\| x_{n} \\
-p\left\|+\left(1-\alpha_{n}\right) \lambda \beta_{n}\right\| p \| \leq \max \left\{\left\|x_{n}-p\right\|,\|p\|\right\} \\
\vdots \\
\leq \max \left\{\left\|x_{0}-p\right\|,\|p\|\right\} .
\end{gathered}
$$

It follows that $\left\{x_{n}\right\}$ is bounded, and so are $\left\{P_{C}(I-\lambda(\nabla g+\right.$ $\left.\left.\left.\beta_{n} I\right)\right) x_{n}\right\}$ and $\left\{P_{C}(I-\lambda \nabla g) x_{n}\right\}$.

Step 2. We will show that $\lim _{n \rightarrow \infty}\left\|x_{n}-P_{C}(I-\lambda \nabla g) x_{n}\right\|=0$. Since,

$$
\begin{aligned}
& \left\|x_{n+1}-P_{C}(I-\lambda \nabla g) x_{n}\right\|=\| \alpha_{n} x_{n}+\left(1-\alpha_{n}\right) \\
& \quad \cdot P_{C}\left(I-\lambda\left(\nabla g+\beta_{n} I\right)\right) x_{n}-P_{C}(I-\lambda \nabla g) x_{n} \| \\
& =\| \alpha_{n} x_{n}+\left(1-\alpha_{n}\right) P_{C}\left(I-\lambda\left(\nabla g+\beta_{n} I\right)\right) x_{n} \\
& \quad-\left(\alpha_{n}+\left(1-\alpha_{n}\right)\right) P_{C}(I-\lambda \nabla g) x_{n} \| \\
& =\| \alpha_{n}\left(x_{n}-P_{C}(I-\lambda \nabla g) x_{n}\right)+\left(1-\alpha_{n}\right) \\
& \cdot\left(P_{C}\left(I-\lambda\left(\nabla g+\beta_{n} I\right)\right) x_{n}-P_{C}(I-\lambda \nabla g) x_{n}\right) \| \\
& \leq \alpha_{n}\left\|x_{n}-P_{C}(I-\lambda \nabla g) x_{n}\right\|+\left(1-\alpha_{n}\right) \\
& \cdot \| P_{C}\left(I-\lambda\left(\nabla g+\beta_{n} I\right)\right) x_{n} \\
& -P_{C}(I-\lambda \nabla g) x_{n}\left\|\leq \alpha_{n}\right\| x_{n}-P_{C}(I-\lambda \nabla g) x_{n} \| \\
& +\left(1-\alpha_{n}\right)\left\|\left(I-\lambda\left(\nabla g+\beta_{n} I\right)\right) x_{n}-(I-\lambda \nabla g) x_{n}\right\| \\
& =\alpha_{n}\left\|x_{n}-P_{C}(I-\lambda \nabla g) x_{n}\right\|+\left(1-\alpha_{n}\right) \lambda \beta_{n}\left\|x_{n}\right\| .
\end{aligned}
$$

Therefore, by conditions (i) and (ii), we have

$$
\lim _{n \longrightarrow \infty}\left\|x_{n+1}-P_{C}(I-\lambda \nabla g) x_{n}\right\|=0 .
$$

Since, $\nabla g$ is $1 / L$-ism, then we have

$$
\begin{aligned}
&\left\|P_{C}(I-\lambda \nabla g) x-P_{C}(I-\lambda \nabla g) y\right\|^{2} \\
& \leq\|(I-\lambda \nabla g) x-(I-\lambda \nabla g) y\|^{2} \\
&=\|(x-y)-\lambda(\nabla g(x)-\nabla g(y))\|^{2} \\
&=\|x-y\|^{2}-2 \lambda\langle x-y, \nabla g(x)-\nabla g(y)\rangle \\
&+\lambda^{2}\|\nabla g(x)-\nabla g(y)\|^{2} \\
& \leq\|x-y\|^{2}-\frac{2 \lambda}{L}\|\nabla g(x)-\nabla g(y)\|^{2} \\
&+\lambda^{2}\|\nabla g(x)-\nabla g(y)\|^{2}
\end{aligned}
$$

$$
\begin{aligned}
& =\|x-y\|^{2}-\lambda\left(\frac{2}{L}-\lambda\right)\|\nabla g(x)-\nabla g(y)\|^{2} \\
& \leq\|x-y\|^{2} .
\end{aligned}
$$

Hence,

$$
\left\|P_{C}(I-\lambda \nabla g) x-P_{C}(I-\lambda \nabla g) y\right\| \leq\|x-y\|,
$$

that is, $P_{C}(I-\lambda \nabla g)$ is a nonexpansive. Therefore, by (36) and NST-condition (II) in Lemma 8, we have

$$
\begin{aligned}
& \lim _{n \longrightarrow \infty}\left\|\left(I-P_{C}(I-\lambda \nabla g)\right) x_{n}\right\| \\
& \quad=\lim _{n \longrightarrow \infty}\left\|x_{n}-P_{C}(I-\lambda \nabla g) x_{n}\right\|=0 .
\end{aligned}
$$

Step 3. Let $q \in \mathcal{U}$. Since, $\mathscr{U}$ is a singleton set, we have

$$
\langle-q, q-y\rangle \geq 0, \quad \forall y \in \mathcal{U} .
$$

Therefore, by Lemma 3 , we have $q=P_{\mathcal{U}}(0)$. We will show that $\lim \sup _{n \rightarrow \infty}\left\langle-q, x_{n+1}-q\right\rangle \leq 0$. From (10) we have

$$
q=P_{C}(I-\lambda \nabla g) q .
$$

Since $\left\{x_{n}\right\}$ is bounded, we consider a subsequence $\left\{x_{n_{i j}}\right\}$ of $\left\{x_{n_{i}}\right\}$; there exists a subsequence $\left\{x_{n_{i_{j}}}\right\}$ of $\left\{x_{n_{i}}\right\}$ which converges weakly to $z$. It follows by the demiclosedness to the zero in Lemma 9 and (39) that $z=P_{C}(I-\lambda \nabla g) z$. So, by (10) we have $z \in \mathscr{U}$ (indeed, $z=q$ ). Therefore, by (40), we have

$$
\begin{aligned}
& \limsup _{n \rightarrow \infty}\left\langle-q, x_{n+1}-q\right\rangle=\limsup _{j \rightarrow \infty}\left\langle-q, x_{n_{i_{j}}+1}-q\right\rangle \\
& \quad=\max \left\{\langle-q, z-q\rangle: \text { for all } x_{n_{i_{j}}}-z \text { as } j \longrightarrow \infty\right\}
\end{aligned}
$$$$
\leq 0 .
$$

Step 4. We will show that $\left\{x_{n}\right\}$ converges strongly to $q$. By (30), Lemma 5, condition (i), and the linearity orthogonal projection of $P_{C}$, we have

$$
\begin{aligned}
& \left\|x_{n+1}-q\right\|^{2}=\| \alpha_{n} x_{n}+\left(1-\alpha_{n}\right) P_{C}\left(I-\lambda\left(\nabla g+\beta_{n} I\right)\right) \\
& \cdot x_{n}-q\left\|^{2}=\right\| \alpha_{n} x_{n}+\left(1-\alpha_{n}\right) P_{C}(I \\
& \left.-\lambda\left(\nabla g+\beta_{n} I\right)\right) x_{n}-\left(\alpha_{n}+\left(1-\alpha_{n}\right)\right) q \|^{2} \\
& =\| \alpha_{n}\left(x_{n}-q\right)+\left(1-\alpha_{n}\right) \\
& \cdot\left(P_{C}\left(I-\lambda\left(\nabla g+\beta_{n} I\right)\right) x_{n}-q\right)\left\|^{2} \leq \alpha_{n}\right\| x_{n}-q \|^{2} \\
& \quad+\left(1-\alpha_{n}\right)\left\|P_{C}\left(I-\lambda\left(\nabla g+\beta_{n} I\right)\right) x_{n}-q\right\|^{2} \\
& =\alpha_{n}\left\|x_{n}-q\right\|^{2}+\left(1-\alpha_{n}\right)\left\langle P_{C}\left(I-\lambda\left(\nabla g+\beta_{n} I\right)\right)\right. \\
& \left.\quad \cdot x_{n}-q, P_{C}\left(I-\lambda\left(\nabla g+\beta_{n} I\right)\right) x_{n}-q\right\rangle=\alpha_{n} \| x_{n}
\end{aligned}
$$




$$
\begin{aligned}
& -q \|^{2}+\left(1-\alpha_{n}\right)\left\langle P_{C}\left(I-\lambda\left(\nabla g+\beta_{n} I\right)\right) x_{n}-q\right. \text {, } \\
& \left.\frac{x_{n+1}-\alpha_{n} x_{n}}{1-\alpha_{n}}-q\right\rangle=\alpha_{n}\left\|x_{n}-q\right\|^{2}+\left(1-\alpha_{n}\right) \\
& \cdot\left(\frac { 1 } { 1 - \alpha _ { n } } \left\langleP_{C}\left(I-\lambda\left(\nabla g+\beta_{n} I\right)\right) x_{n}-q, x_{n+1}\right.\right. \\
& -q\rangle+\frac{\alpha_{n}}{1-\alpha_{n}}\left\langle P_{C}\left(I-\lambda\left(\nabla g+\beta_{n} I\right)\right) x_{n}-q, q\right. \\
& \left.\left.-x_{n}\right\rangle\right)=\alpha_{n}\left\|x_{n}-q\right\|^{2}+\left\langle P_{C}\left(I-\lambda\left(\nabla g+\beta_{n} I\right)\right)\right. \\
& \left.\cdot x_{n}-P_{C}(I-\lambda \nabla g) q, x_{n+1}-q\right\rangle+\alpha_{n}\left\langle P_{C}(I\right. \\
& \left.\left.-\lambda\left(\nabla g+\beta_{n} I\right)\right) x_{n}-q, q-x_{n}\right\rangle \leq \alpha_{n}\left\|x_{n}-q\right\|^{2} \\
& +\left\langle P_{C}\left(I-\lambda\left(\nabla g+\beta_{n} I\right)\right) x_{n}-P_{C}(I\right. \\
& \left.\left.-\lambda\left(\nabla g+\beta_{n} I\right)\right) q, x_{n+1}-q\right\rangle+\left\langle P_{C}(I\right. \\
& \left.\left.-\lambda\left(\nabla g+\beta_{n} I\right)\right) q-P_{C}(I-\lambda \nabla g) q, x_{n+1}-q\right\rangle \\
& +\alpha_{n}\left\|P_{C}\left(I-\lambda\left(\nabla g+\beta_{n} I\right)\right) x_{n}-q\right\| \cdot\left\|q-x_{n}\right\| \\
& \leq \alpha_{n}\left\|x_{n}-q\right\|^{2}+\| P_{C}\left(I-\lambda\left(\nabla g+\beta_{n} I\right)\right) x_{n} \\
& -P_{C}\left(I-\lambda\left(\nabla g+\beta_{n} I\right)\right) q\|\cdot\| x_{n+1}-q \|+\lambda \beta_{n}\langle-q \text {, } \\
& \left.x_{n+1}-q\right\rangle+\alpha_{n}\left\|P_{C}\left(I-\lambda\left(\nabla g+\beta_{n} I\right)\right) x_{n}-q\right\| \cdot \| q \\
& -x_{n}\left\|\leq \beta_{n}^{m}\right\| x_{n}-q\left\|^{2}+\left(1-\lambda \beta_{n}\right)\right\| x_{n}-q\|\cdot\| x_{n+1} \\
& -q\left\|+\lambda \beta_{n}\left\langle-q, x_{n+1}-q\right\rangle+\beta_{n}^{m}\right\| P_{C}(I \\
& \left.-\lambda\left(\nabla g+\beta_{n} I\right)\right) x_{n}-q\|\cdot\| q-x_{n}\left\|=\beta_{n}^{m}\right\| x_{n}-q \|^{2} \\
& +\frac{1-\lambda \beta_{n}}{2}\left(\left\|x_{n}-q\right\|^{2}+\left\|x_{n+1}-q\right\|^{2}-\left(\left\|x_{n}-q\right\|\right.\right. \\
& \left.\left.-\left\|x_{n+1}-q\right\|\right)^{2}\right)+\lambda \beta_{n}\left\langle-q, x_{n+1}-q\right\rangle+\beta_{n}^{m} \| P_{C}(I \\
& \left.-\lambda\left(\nabla g+\beta_{n} I\right)\right) x_{n}-q\|\cdot\| q-x_{n}\left\|\leq \beta_{n}^{m}\right\| x_{n}-q \|^{2} \\
& +\frac{1-\lambda \beta_{n}}{2}\left\|x_{n}-q\right\|^{2}+\frac{1}{2}\left\|x_{n+1}-q\right\|^{2}+\lambda \beta_{n}\langle-q \text {, } \\
& \left.x_{n+1}-q\right\rangle+\beta_{n}^{m}\left\|P_{C}\left(I-\lambda\left(\nabla g+\beta_{n} I\right)\right) x_{n}-q\right\| \cdot \| q \\
& -x_{n} \|
\end{aligned}
$$

and, therefore,

$$
\begin{aligned}
& \left\|x_{n+1}-q\right\|^{2} \leq 2 \beta_{n}^{m}\left\|x_{n}-q\right\|^{2}+\left(1-\lambda \beta_{n}\right)\left\|x_{n}-q\right\|^{2} \\
& \quad+2 \lambda \beta_{n}\left\langle-q, x_{n+1}-q\right\rangle \\
& \quad+2 \beta_{n}^{m}\left\|P_{C}\left(I-\lambda\left(\nabla g+\beta_{n} I\right)\right) x_{n}-q\right\| \cdot\left\|q-x_{n}\right\| \\
& \quad=\left(1-\lambda \beta_{n}\right)\left\|x_{n}-q\right\|^{2}+\lambda \beta_{n}\left(\frac{2 \beta_{n}^{m-1}}{\lambda}\left\|x_{n}-q\right\|^{2}\right.
\end{aligned}
$$

$$
\begin{aligned}
& +2\left\langle-q, x_{n+1}-q\right\rangle \\
& +\frac{2 \beta_{n}^{m-1}}{\lambda}\left\|P_{C}\left(I-\lambda\left(\nabla g+\beta_{n} I\right)\right) x_{n}-q\right\| \\
& \left.\cdot\left\|q-x_{n}\right\|\right)=\left(1-\gamma_{n}\right)\left\|x_{n}-q\right\|^{2}+\gamma_{n} \delta_{n}
\end{aligned}
$$

where $\gamma_{n}=\lambda \beta_{n}$ and

$$
\begin{aligned}
\delta_{n}= & \frac{2 \beta_{n}^{m-1}}{\lambda}\left\|x_{n}-q\right\|^{2}+2\left\langle-q, x_{n+1}-q\right\rangle \\
& +\frac{2 \beta_{n}^{m-1}}{\lambda}\left\|P_{C}\left(I-\lambda\left(\nabla g+\beta_{n} I\right)\right) x_{n}-q\right\| \\
& \cdot\left\|q-x_{n}\right\| .
\end{aligned}
$$

It is easy to see that $\sum_{n=0}^{\infty} \gamma_{n}=\infty$ and $\limsup _{n \rightarrow \infty} \delta_{n} \leq 0$. Therefore, by Lemma 10, we obtain $\left\{x_{n}\right\}$ converges strongly to $q$. This completes the proof.

Notice that when $\alpha_{n}=0$ for all $n=0,1,2, \ldots$ then the result of Theorem 11 can be reduced into the result of Tian and Zhang [26] without the control condition $\sum_{n=0}^{\infty}\left|\beta_{n+1}-\beta_{n}\right|<$ $\infty$ as follows.

Corollary 12 (see [26]). Let $C$ be a nonempty closed convex subset of a real Hilbert space $H$ and $g: C \longrightarrow \mathbb{R}$ be a strictly real-valued convex function such that the gradient $\nabla g$ is $1 / L$ ism with $L>0$. Assume that $\mathcal{U} \neq \emptyset$ and let $\left\{x_{n}\right\} \subset C$ be a sequence generated by

$$
\begin{aligned}
x_{0} & \in C, \\
x_{n+1} & =P_{C}\left(I-\lambda\left(\nabla g+\beta_{n} I\right)\right) x_{n}, \quad \forall n=0,1,2, \ldots,
\end{aligned}
$$

where $\lambda \in(0,2 /(2+L))$ and $\left\{\beta_{n}\right\} \subset(0,1)$ satisfies the following conditions: $\lim _{n \rightarrow \infty} \beta_{n}=0$ and $\sum_{n=0}^{\infty} \beta_{n}=\infty$, then the sequence $\left\{x_{n}\right\}$ converges strongly to $q \in \mathcal{U}$, which is the unique minimizer of the CCMP (1).

\section{Applications}

Let $C$ and $Q$ be closed convex subsets of real Hilbert spaces $H_{1}$ and $H_{2}$, respectively, and $A: H_{1} \longrightarrow H_{2}$ be a bounded linear operator. We now consider the split feasibility problem (SFP) which introduced in 1994 by Censor and Elfving [13], where this problem is to find an element $q \in C$ such that $A q \in Q$. Define the convex function $g: C \longrightarrow \mathbb{R}$ as follows:

$$
g(x)=\frac{1}{2}\left\|A x-P_{\mathrm{Q}} A x\right\|^{2} .
$$

It follows by Lemma 7 that the gradient of $g$ as $\nabla g=A^{*}(I-$ $\left.P_{\mathrm{Q}}\right) A$ where $A^{*}$ is the adjoint operator of $A$, and $\nabla g$ is $1 /\|A\|^{2}$ ism. We have the consequence results as follows.

Theorem 13. Let $C$ and $Q$ be closed convex subsets of real Hilbert spaces $H_{1}$ and $H_{2}$, respectively, and let $A: H_{1} \longrightarrow$ 
$\mathrm{H}_{2}$ be a bounded linear operator. Suppose that the SFP has a nonempty solution. Let $\left\{x_{n}\right\} \subset C$ be a sequence generated by

$$
\begin{aligned}
& x_{0} \in \mathrm{C}, \\
& \begin{aligned}
x_{n+1} & \\
= & \alpha_{n} x_{n} \\
& +\left(1-\alpha_{n}\right) P_{C}\left(I-\lambda\left(A^{*}\left(I-P_{\mathrm{Q}}\right) A+\beta_{n} I\right)\right) x_{n}, \\
& \forall n=0,1,2, \ldots,
\end{aligned}
\end{aligned}
$$

where $\lambda \in\left(0,2 /\left(2+\|A\|^{2}\right)\right)$ and $\left\{\alpha_{n}\right\} \subset[0,1),\left\{\beta_{n}\right\} \subset(0,1)$ satisfy the following conditions:

(i) $\alpha_{n} \leq \beta_{n}^{m}$ such that $m>1$ for all $n=0,1,2, \ldots$,

(ii) $\lim _{n \rightarrow \infty} \beta_{n}=0$ and $\sum_{n=0}^{\infty} \beta_{n}=\infty$,

then the sequence $\left\{x_{n}\right\}$ converges strongly to $q$, which is the unique minimizer of the minimum-norm solution of the SFP.

Corollary 14. Let $C$ and $Q$ be closed convex subsets of real Hilbert spaces $H_{1}$ and $H_{2}$, respectively, and let $A: H_{1} \longrightarrow$ $\mathrm{H}_{2}$ be a bounded linear operator. Suppose that the SFP has a nonempty solution. Let $\left\{x_{n}\right\} \subset C$ be a sequence generated by

$$
\begin{aligned}
& x_{0} \in C, \\
& x_{n+1}=P_{C}\left(I-\lambda\left(A^{*}\left(I-P_{Q}\right) A+\beta_{n} I\right)\right) x_{n}, \\
& \forall n=0,1,2, \ldots,
\end{aligned}
$$

where $\lambda \in\left(0,2 /\left(2+\|A\|^{2}\right)\right)$ and $\left\{\beta_{n}\right\} \subset(0,1)$ satisfies the following conditions: $\lim _{n \longrightarrow \infty} \beta_{n}=0$ and $\sum_{n=0}^{\infty} \beta_{n}=\infty$, then the sequence $\left\{x_{n}\right\}$ converges strongly to $q$, which is the unique minimizer of the minimum-norm solution of the SFP.

\section{Data Availability}

In order of the feasible solution, all algorithms of the iterations have to compute many inner iterations to find the appropriate result, and stack overflow often occurs in which a computer program makes too many subroutine calls and its call stack runs out of space when the parameters of iterations have using many stack arrays to compute the feasible solution.

To avoid the stack overflow, we introduce how to do the mathematical programming without using the stack arrays of its parameters for solving the SFP of the algorithm in Corollary 14. Indeed, the situation of the stack overflow may have occurred from calculating the floating point numbers or the significant decimal digits; to avoid it we ought to be careful of that by always using digit precision command such as the command $N$ [expr $]$ in Mathematica, and the command digits(num_) in Matlab, and also define all matrix in the regular command type without using the matrix palette to avoid it.

Some mathematical software has a command to give the total number of seconds of CPU time used and the total number of seconds since the beginning of computation in the session such as the commands TimeUsed[] and AbsoluteTime[] in Mathematica, respectively, and the commands cputime and tic/toc in Matlab, respectively.

We now give the formulation of orthogonal projection $P_{C}$ where $C$ is a simply closed convex sets as follows, and in the case that $C$ is not a simply closed convex sets, for instance, $C$ is a halfspace, we can found more the formulation in [33].

Proposition 15. For $x \in \mathbb{R}^{N}$ we have

(i) if $C=\mathbb{R}^{N}$ then $P_{C} x=x$,

(ii) if $C=\{b\}$ such that $b \in \mathbb{R}^{M}$ then $P_{C} x=b$,

(iii) if $C=\left\{x \in \mathbb{R}^{N}:\|x\|_{2} \leq \rho, \rho>0\right\}$ then

$$
P_{C} x= \begin{cases}\frac{\rho x}{\|x\|_{2}}, & x \notin C, \\ x, & x \in C .\end{cases}
$$

Proof. Obviously, the results (i) and (ii) hold by the definition of orthogonal projection of $P_{C}$, and the result (iii) also holds by the normal vector of the boundary points set of $C$.

We are ready to introduce how to do the mathematical programming without using the stack arrays of its parameters for solving the SFP of the algorithm in Corollary 14 as follows. Suppose that the SFP has the unique consistent. Taking $H_{1}=$ $\left(\mathbb{R}^{N},\|\cdot\|_{2}\right)$ and $H_{2}=\left(\mathbb{R}^{M},\|\cdot\|_{2}\right)$ into Corollary 14. Let the sets $C$ and $Q$, the operator $A$, the sequence $\left\{x_{n}\right\}$, and the parameters $\left\{\beta_{n}\right\}, \lambda$ satisfy the conditions in Corollary 14. We have that $\left\{x_{n}\right\}$ is a convergent sequence, and so it is a Cauchy sequence. Hence, we can choose the stopping criteria $\epsilon>0$ which satisfies $\left\|x_{n+1}-x_{n}\right\|_{2}<\epsilon$ for stopping the program, and also the approximate solution refers to the last iteration. Steps of the mathematical programming of the algorithm in Corollary 14 are shown as follows:

Mathematical programming for the split feasibility problem

Finding the solution of an augmented matrix equation $A_{M \times N} X_{N \times 1}=B_{M \times 1}$.

Step 1. Declare of all parameters $A_{M \times N}, B_{M \times 1}$, the starting point $x \operatorname{Start}_{N \times 1}$ and $\epsilon$.

We set $M=3, N=3$ and $b=B_{M \times 1}$.

The example of the commands in Mathematica is shown as follows.

$A=\{\{1,2,-1\},\{1,1,-1\},\{1,-2,1\}\} ; b=\{\{1\},\{0\},\{3\}\} ;$

$x$ Start $=\{\{0\},\{0\},\{0\}\} ; \epsilon=10^{-6}$;

Step 2. Define the formulations of the orthogonal projections of $P_{C}$ and $P_{Q}$ where

$$
\begin{aligned}
& C=\left\{x \in \mathbb{R}^{N}:\|x\|_{2} \leq \rho, \rho>0\right\}, \\
& Q=\left\{x \in \mathbb{R}^{M}:\|x\|_{2} \leq \sigma, \sigma>0\right\} .
\end{aligned}
$$

If we choose $C=\mathbb{R}^{N}$ and $Q=\{b\}$ such that $b \in \mathbb{R}^{M}$ then the orthogonal projections of $P_{C}$ and $P_{Q}$ are easy to calculated, 
and, hence, we do not need to define its formulations in this step, and we can put directly its formulations to process.

The example of the commands in Mathematica is shown as follows.

$\operatorname{Pc}\left[u_{-}\right]:=\operatorname{If}[\operatorname{Norm}[u]>\rho, \rho u /(\operatorname{Norm}[u] / / N), u] ;$ $P q\left[u_{-}\right]:=\operatorname{If}[\operatorname{Norm}[u]>\sigma, \sigma u /(\operatorname{Norm}[u] / / N), u]$;

Step 3. Set the starting index $n=0$ and fix parameter $\lambda \epsilon$ $\left(0,2 /\left(2+\|A\|^{2}\right)\right)$. If the parameter $\lambda$ is not a fix number such that it is a sequence, then we must lie it in the while loop of step 4 .

We set $\lambda=2 /\left(\left(2+\|A\|^{2}\right)+10^{2}\right)$.

The example of the commands in Mathematica is shown as follows.

$$
n=0 ; \lambda=2 /\left(\left(2+(\operatorname{Norm}[A] / / N)^{2}\right)+10^{2}\right)
$$

Step 4. Start to calculate the iterations of the sequence $\left\{x_{n}\right\}$ such that

$$
x_{n+1}=P_{C}\left(I-\lambda\left(A^{*}\left(I-P_{\mathrm{Q}}\right) A+\beta_{n} I\right)\right) x_{n}
$$

using the while loop. Set the parameter $\left\{\beta_{n}\right\} \subset(0,1)$ for all $n=0,1,2, \ldots$ into the while loop such that it satisfies the following conditions: $\lim _{n \rightarrow \infty} \beta_{n}=0$ and $\sum_{n=0}^{\infty} \beta_{n}=\infty$. If $\left\|x_{n+1}-x_{n}\right\|_{2}<\epsilon$ then we break the while loop for approximate feasible solution, which is referred to in the last iteration.

It well known that, in the case of finite-dimensional real space, $A^{*}=A^{T}$ where $A^{T}$ stands for matrix transposition of $A$, and, hence, the algorithm in Corollary 14 can be reduced to

$$
x_{n+1}= \begin{cases}P_{C}\left(x_{n}-\lambda A^{T} A x_{n}+\lambda A^{T} P_{Q} A x_{n}-\lambda \beta_{n} x_{n}\right) & \text { for the closed balls } C \text { and } Q, \\ P_{C}\left(x_{n}-\lambda A^{T} A x_{n}+\lambda A^{T} b-\lambda \beta_{n} x_{n}\right) & \text { for the closed ball } C \text { and } Q=\{b\}, \\ x_{n}-\lambda A^{T} A x_{n}+\lambda A^{T} b-\lambda \beta_{n} x_{n} & \text { for } C=\mathbb{R}^{N} \text { and } Q=\{b\}\end{cases}
$$

where $\mathrm{b} \in \mathbb{R}^{M}$. We set $\beta_{n}=1 /(n+2)$ for all $n=0,1,2, \ldots$ and instead of $x_{n}$ and $x_{n+1}$ with the variables $x$ Old and $x$ New, respectively, and also instead of $\beta_{n}$ with $\beta$ in the while loop for avoidance using stack arrays of the parameters.

The example of the commands in Mathematica is shown as follows.

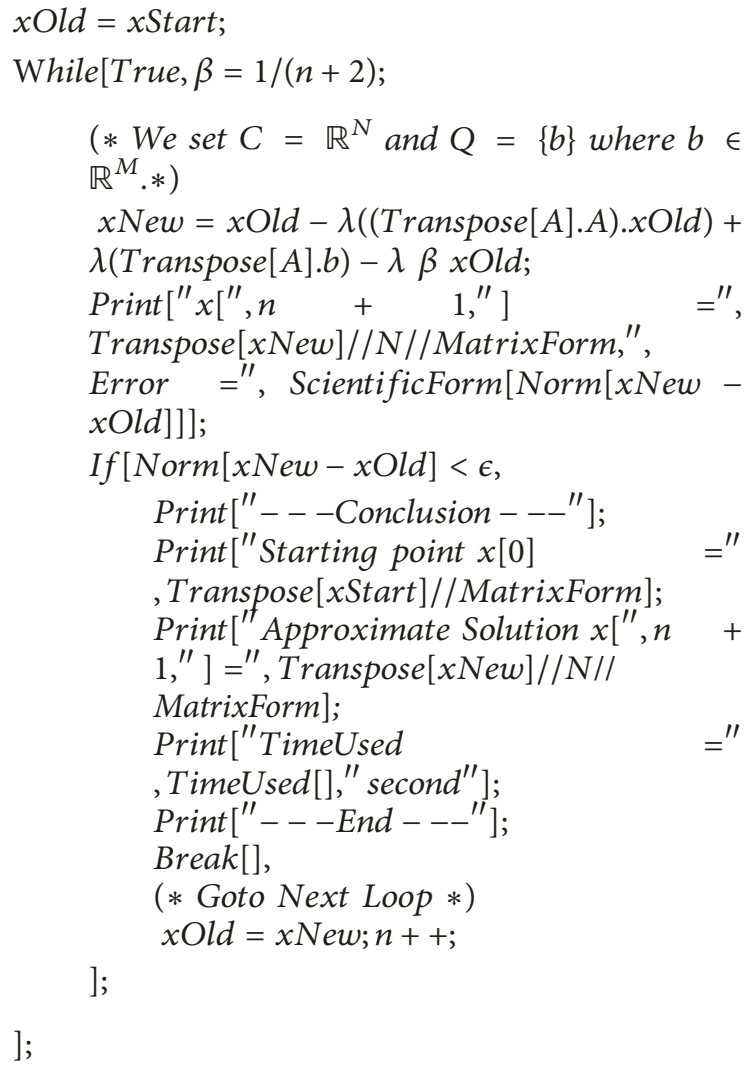

Step 5. Clear memory of the system.

The example of the command in Mathematica is shown as follows.

Quit[];

\section{Numerical Results}

In this section, we give some insight into the behavior of the algorithm presented in Corollary 14 . We implemented them in Mathematica to solve and run on a computer Intel(R) Core (TM) i3 processor $2.00 \mathrm{GHz}$. We use $\left\|x_{n+1}-x_{n}\right\|_{2}<\epsilon$ as the stopping criteria.

Throughout the computational experiments, the parameters used in those algorithms were sets as $\epsilon=10^{-6}, \beta_{n}=$ $1 /(n+2)$ and $\lambda=2 /\left(\left(2+\|A\|^{2}\right)+10^{2}\right)$ for all $n=$ $0,1,2, \ldots$, where $A$ is a bounded linear operator. In the results report below, all CPU times reported are in seconds. The approximate solution is referred to the last iteration.

Example 1. Find the solution of linear equation system as follows:

$$
\begin{gathered}
x+2 y-z=1, \\
x+y-z=0, \\
x-2 y+z=3,
\end{gathered}
$$

where $x, y, z \in \mathbb{R}$.

$$
\text { Let } H_{1}=H_{2}=\left(\mathbb{R}^{3},\|\cdot\|_{2}\right) \text {. Take } A=\left(\begin{array}{ccc}
1 & 2 & -1 \\
1 & 1 & -1 \\
1 & -2 & 1
\end{array}\right), C=\mathbb{R}^{3}
$$
and $Q=\left\{b: b=(1,0,3)^{T}\right\}$ into Corollary 14 . We have chosen $x_{0} \in C$ arbitrarily and 
TABLE 1: Results for Example 1 using algorithm in Corollary 14.

\begin{tabular}{lccc}
\hline Starting points $x_{0}$ & Number of iterations & CPU(s) & Approximate solution q \\
\hline$(0,0,0)^{T}$ & 6015 & 2.393 & $(1.999190,0.997529,2.995430)^{T}$ \\
$(1,1,1)^{T}$ & 5986 & 2.293 & $(1.999180,0.997518,2.995410)^{T}$ \\
$(2,2,2)^{T}$ & 5954 & 2.333 & $(1.999180,0.997506,2.995380)^{T}$ \\
$(2,3,1)^{T}$ & 5963 & 2.543 & $(1.999180,0.997509,2.995390)^{T}$ \\
$(3,2,1)^{T}$ & 5970 & 2.533 & $(1.999180,0.997512,2.995400)^{T}$ \\
\hline
\end{tabular}

$$
\begin{aligned}
x_{n+1} & =P_{C}\left(I-\lambda\left(A^{T}\left(I-P_{\mathrm{Q}}\right) A+\beta_{n} I\right)\right) x_{n} \\
& =x_{n}-\lambda A^{T} A x_{n}+\lambda A^{T} P_{\mathrm{Q}} A x_{n}-\lambda \beta_{n} x_{n} \\
& =x_{n}-\lambda A^{T} A x_{n}+\lambda A^{T} b-\lambda \beta_{n} x_{n}
\end{aligned}
$$

where $b=(1,0,3)^{T}$ for all $n=0,1,2, \ldots$ As $n \longrightarrow \infty$, we have $x_{n} \longrightarrow q$ such that $q$ is the our solution. The numerical results are listed in Table 1.

\section{Conclusion}

In this paper, we obtain an iterative scheme using the gradient projection method based on Mann's approximation method for solving the constrained convex minimization problem (CCMP) and also solving the split feasibility problem (SFP) such that another strong convergence theorems for the CCMP and the SFP are obtained.

\section{Data Availability}

The data used to support the findings of this study are included within the article.

\section{Conflicts of Interest}

The authors declare that there are no conflicts of interest regarding the publication of this paper.

\section{Acknowledgments}

The authors would like to acknowledge the Science Achievement Scholarship of Thailand (SAST) and the Faculty of Science, Maejo University, for financial support.

\section{References}

[1] E. S. Levitin and B. T. Polyak, "Constrained minimization methods," Zhurnal Vychislitel'noi Matematiki i Matematicheskoi Fiziki, vol. 6, no. 5, pp. 787-823, 1966.

[2] H.-K. Xu, "Averaged mappings and the gradient-projection algorithm," Journal of Optimization Theory and Applications, vol. 150, no. 2, pp. 360-378, 2011.

[3] P. Kumam, "A new hybrid iterative method for solution of equilibrium problems and fixed point problems for an inverse strongly monotone operator and a nonexpansive mapping," Journal of Applied Mathematics and Computing, vol. 29, no. 12, pp. 263-280, 2009.
[4] Y. Yao and H.-K. Xu, "Iterative methods for finding minimumnorm fixed points of nonexpansive mappings with applications," Journal of Mathematical Programming and Operations Research, vol. 60, no. 6, pp. 645-658, 2011.

[5] S. He and W. Sun, "New hybrid steepest descent algorithms for variational inequalities over the common fixed points set of infinite nonexpansive mappings," WSEAS Transactions on Mathematics, vol. 11, no. 2, pp. 83-92, 2012.

[6] M. Su and H.-K. Xu, "Remarks on the gradient-projection algorithm," Journal of Nonlinear Analysis and Optimization, vol. 1, no. 1, pp. 35-43, 2010.

[7] R.-X. Ni, "Strong convergence of a hybrid projection algorithm for approximation of a common element of three sets in Banach spaces," WSEAS Transactions on Mathematics, vol. 12, no. 3, pp. 296-306, 2013.

[8] P. Kumam, "A hybrid approximation method for equilibrium and fixed point problems for a monotone mapping and a nonexpansive mapping," Nonlinear Analysis: Hybrid Systems, vol. 2, no. 4, pp. 1245-1255, 2008.

[9] P. H. Calamai and J. J. Moré, "Projected gradient methods for linearly constrained problems," Mathematical Programming, vol. 39, no. 1, pp. 93-116, 1987.

[10] F. Wang and H.-K. Xu, "Approximating curve and strong convergence of the CQ algorithm for the split feasibility problem," Journal of Inequalities and Applications, vol. 2010, Article ID 102085, 13 pages, 2010.

[11] J. S. Jung, "Strong convergence of composite iterative methods for equilibrium problems and fixed point problems," Applied Mathematics and Computation, vol. 213, no. 2, pp. 498-505, 2009.

[12] J.S. Jung, "Strong convergence of iterative methods for $k$-strictly pseudo-contractive mappings in Hilbert spaces," Applied Mathematics and Computation, vol. 215, no. 10, pp. 3746-3753, 2010.

[13] Y. Censor and T. Elfving, "A multiprojection algorithm using Bregman projections in a product space," Numerical Algorithms, vol. 8, no. 2-4, pp. 221-239, 1994.

[14] H.-K. Xu, "A variable Krasnosel'skii-Mann algorithm and the multiple-set split feasibility problem," Inverse Problems, vol. 22, no. 6, pp. 2021-2034, 2006.

[15] H.-K. Xu, "Iterative methods for the split feasibility problem in infinite-dimensional Hilbert spaces," Inverse Problems, vol. 26, no. 10, Article ID 105018, 2010.

[16] C. Byrne, "A unified treatment of some iterative algorithms in signal processing and image reconstruction," Inverse Problems, vol. 20, no. 1, pp. 103-120, 2004.

[17] H. Songnian and G. Jun, "Algorithms for finding the minimum norm solution of hierarchical fixed point problems," WSEAS Transactions on Mathematics, vol. 12, no. 3, pp. 317-328, 2013. 
[18] G. Lopez, V. Martin, and H.-K. Xu, "Perturbation techniques for nonexpansive mappings with applications," Nonlinear Analysis: Real World Applications, vol. 10, no. 4, pp. 2369-2383, 2009.

[19] L.-C. Ceng, Q. H. Ansari, and J.-C. Yao, "Extragradientprojection method for solving constrained convex minimization problems," Numerical Algebra, Control and Optimization, vol. 1, no. 3, pp. 341-359, 2011.

[20] Y. Yao, S. M. Kang, W. Jigang, and P.-X. Yang, "A Regularized Gradient Projection Method for the Minimization Problem," Journal of Applied Mathematics, vol. 2012, Article ID 259813, 9 pages, 2012.

[21] Y. Yao, Y.-C. Liou, and C.-F. Wen, "Variant gradient projection methods for the minimization problems," Abstract and Applied Analysis, vol. 2012, Article ID 792078, 16 pages, 2012.

[22] M. Tian and M. Li, "A general iterative method for constrained convex minimization problems in hilbert spaces," WSEAS Transactions on Mathematics, vol. 13, pp. 271-281, 2014.

[23] G. Cai and Y. Shehu, "An iterative algorithm for fixed point problem and convex minimization problem with applications," Fixed Point Theory and Applications, vol. 2015, no. 7, 17 pages, 2015.

[24] C. D. Enyi and M. E. Soh, "Modified gradient-projection algorithm for solving convex minimization problem in Hilbert spaces," IAENG International Journal of Applied Mathematics, vol. 44, no. 3, 7 pages, 2014.

[25] Y. Wu and L. Shi, "Projection and contraction methods for constrained convex minimization problem and the zero points of maximal monotone operator," Journal of Nonlinear Sciences and Applications. JNSA, vol. 10, no. 2, pp. 637-646, 2017.

[26] M. Tian and H.-F. Zhang, "Regularized gradient-projection methods for finding the minimum-norm solution of the constrained convex minimization problem," Journal of Inequalities and Applications, vol. 2017, no. 13, 12 pages, 2017.

[27] W. Takahashi, Introduction to Nonlinear and Convex Analysis, Yokohama Publishers, Yokohama, Japan, 2009.

[28] J. F. Tang, S. S. Chang, and F. Yuan, "A strong convergence theorem for equilibrium problems and split feasibility problems in Hilbert spaces," Fixed Point Theory and Applications, vol. 2014, no. 36, 16 pages, 2014.

[29] K. Nakajo, K. Shimoji, and W. Takahashi, "Strong convergence to common fixed points of families of nonexpansive mappings in Banach spaces," Journal of Nonlinear and Convex Analysis. An International Journal, vol. 8, no. 1, pp. 11-34, 2007.

[30] W. Takahashi, Y. Takeuchi, and R. Kubota, "Strong convergence theorems by hybrid methods for families of nonexpansive mappings in Hilbert spaces," Journal of Mathematical Analysis and Applications, vol. 341, no. 1, pp. 276-286, 2008.

[31] H. S. Hundal, "An alternating projection that does not converge in norm," Nonlinear Analysis: Theory, Methods \& Applications, vol. 57, no. 1, pp. 35-61, 2004.

[32] H. K. Xu, "Viscosity approximation methods for nonexpansive mappings," Journal of Mathematical Analysis and Applications, vol. 298, no. 1, pp. 279-291, 2004.

[33] P. Tianchai, "Gradient projection method with a new step size for the split feasibility problem," Journal of Inequalities and Applications, vol. 2018, no. 120, 22 pages, 2018. 


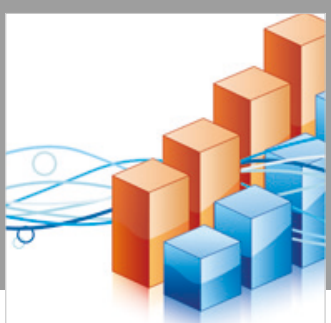

Advances in

Operations Research

\section{-n-m}
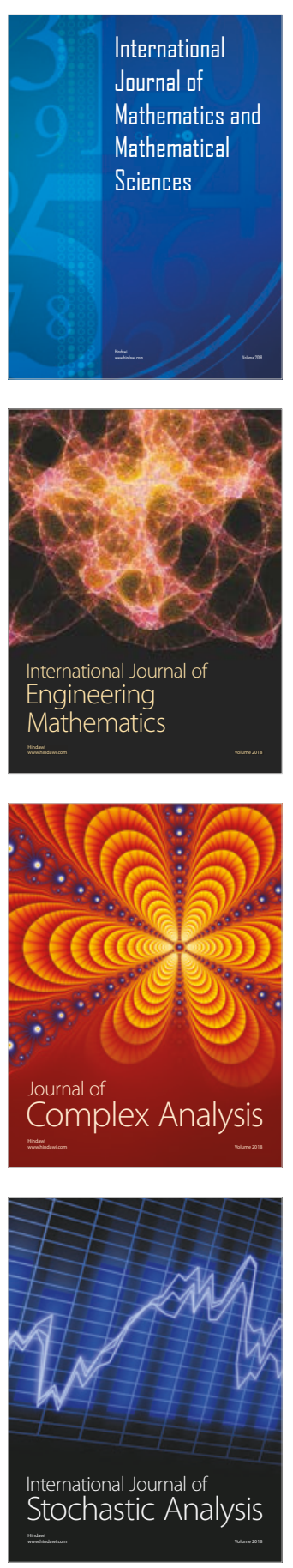
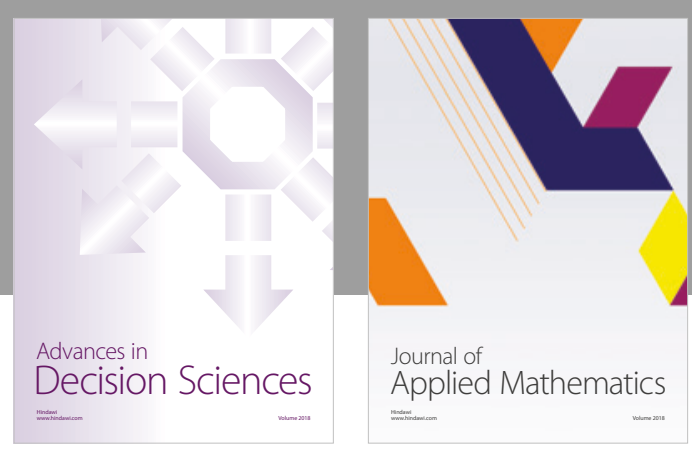

Journal of

Applied Mathematics
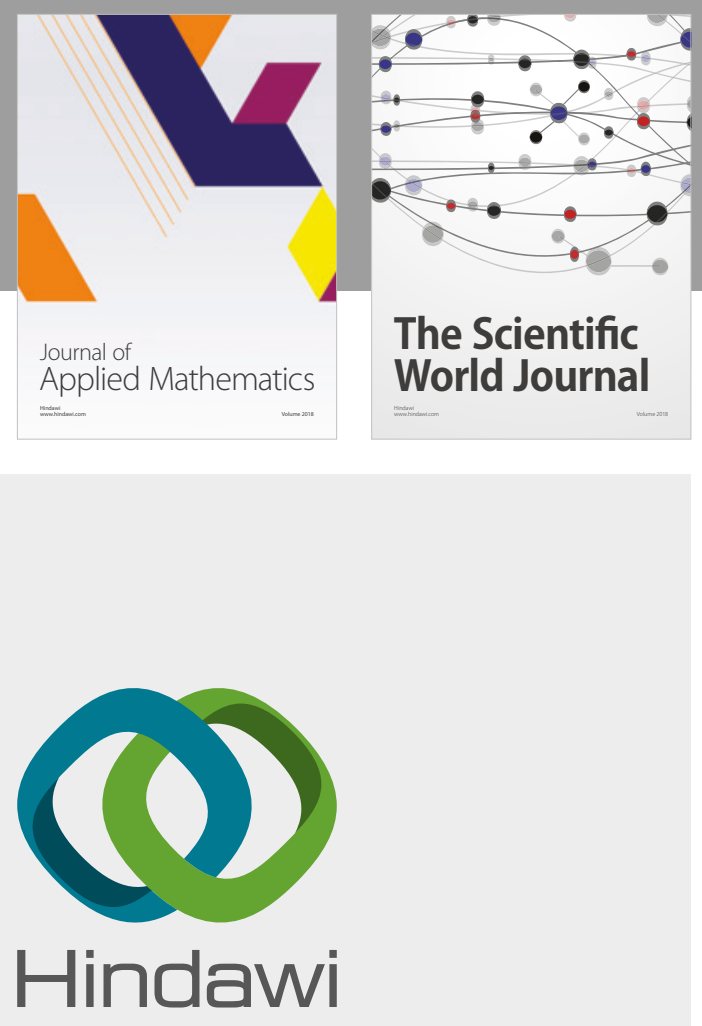

Submit your manuscripts at

www.hindawi.com

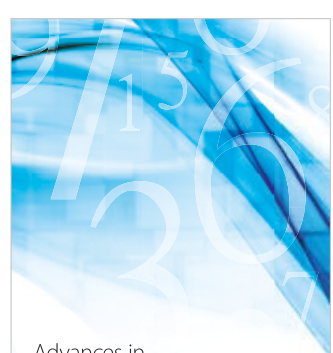

Advances in
Numerical Analysis
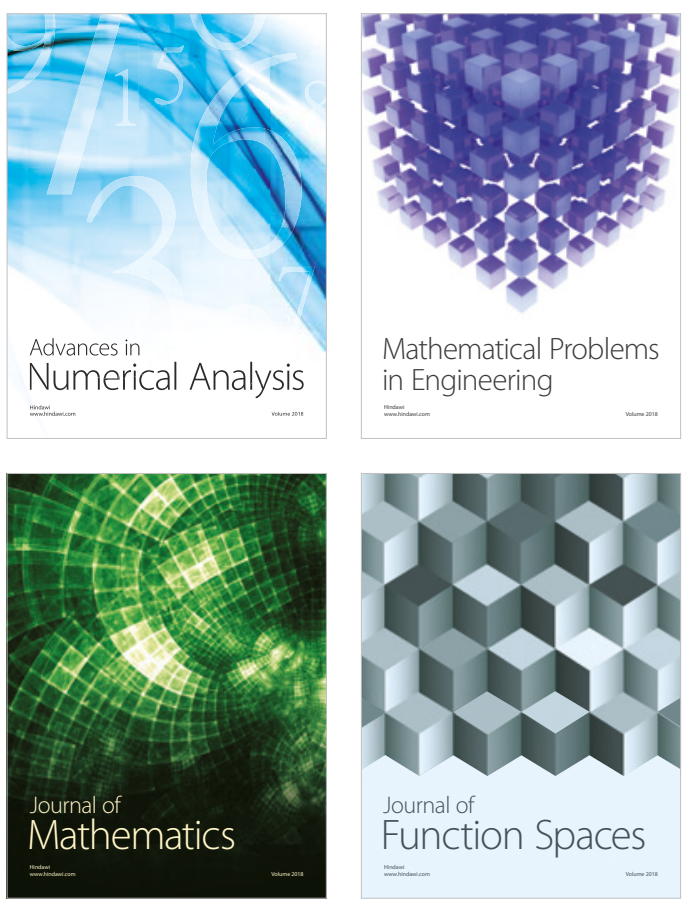

Mathematical Problems in Engineering

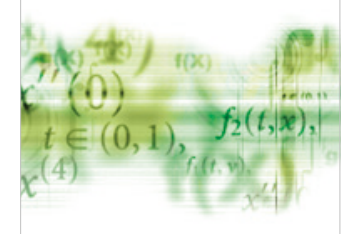

International Journal of

Differential Equations

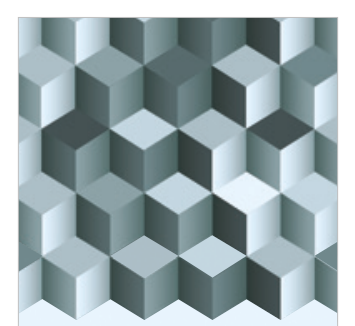

Journal of

Function Spaces

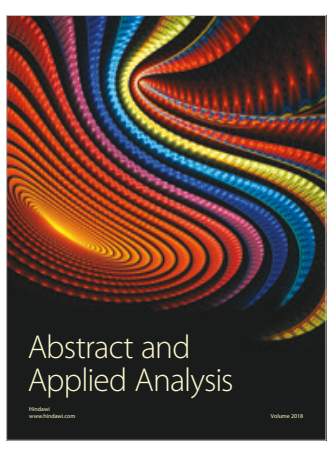

The Scientific

World Journal

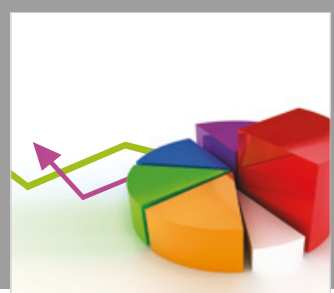

Journal of

Probability and Statistics
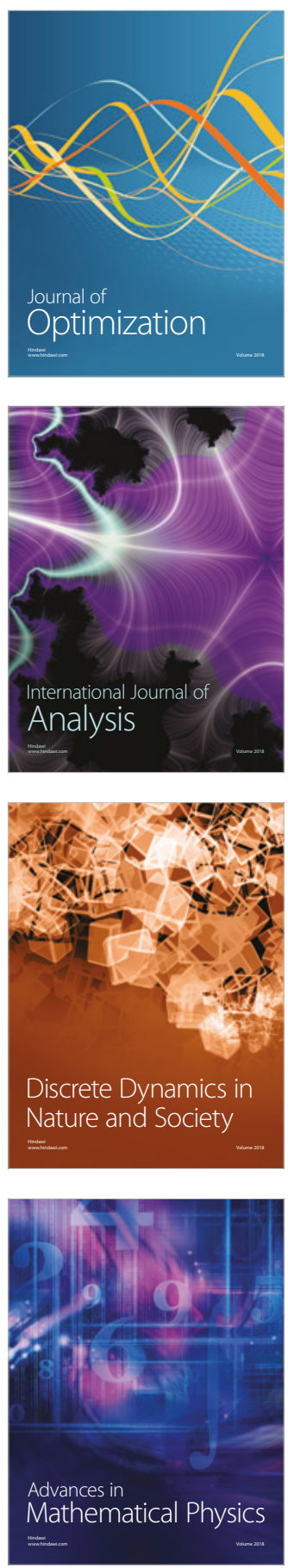